\title{
Epidemiology
}

\section{Epidemic of prosthetic valve endocarditis caused by Staphylococcus epidermidis}

\author{
P J VAN DEN BROEK, A S LAMPE, G A M BERBÉE, J THOMPSON, R P MOUTON
}

\begin{abstract}
In an epidemic of prosthetic valve endocarditis caused by Staphylococcus epidermidis the surgeon was found to be the source of contamination. The probable route was accidental puncture of gloves during operation. During the epidemiological investigation a second cluster of patients contaminated with Staph epidermidis during open heart surgery was found also related to one surgeon. This strain caused no detectable signs or symptoms of infection.
\end{abstract}

Carriage of virulent staph epidermidis has rarely been recognised as a hazard but may have serious consequences.

\section{Introduction}

Staphylococcus epidermidis is an important causative agent in infections of prosthetic heart valves.' Peroperative contamination of the surgical site and the prosthesis with Staph epidermidis and diphtheroides occurs in about $70 \%$ of open heart operations. ${ }^{2}$ Staph epidermidis is normally present on human skin, and it has been suggested that the main route of peroperative contamination by this micro-organism is sedimentation from the air. ${ }^{3}$

We report two clusters of patients whose cultures grew Staph epidermidis after cardiac surgery. Staph epidermidis was cultured from heart-lung machines, blood, a venous catheter, an arterial catheter, a sternal wound, and, in the first cluster of cases, the prosthetic heart valves also. For each cluster a surgeon was found to be the source of the contamination, and it is postulated that accidental puncture of gloves during operation was the route.

\begin{abstract}
Methods
Blood was cultured aerobically in brain-heart infusion broth (Oxoid CM 225) supplemented with haemin, nicotinamide adenine dinucleotide, sodium polyanethole sulphonate, and gelatin; and anaerobically in tryptone soya broth (Oxoid CM 129) supplemented with cysteine, sodium polyanethole sulphonate, agar, and resazurin. Isolation of Staph epidermidis strains was performed after subculture on blood agar. Cardiac valves and surrounding tissues were pulverised in Potter vessels and cultured on the same blood agar media. Identification was based on colony morphology, microscopical features, catalase production, and the absence of coagulase production. In doubtful cases a deoxyribonuclease or thermonuclease test was also done. Antibiotic sensitivities were tested by disc diffusion as
\end{abstract}

University Hospital, Rijnsburgerweg 10, 2333 AA Leiden, The Netherlands P J VAN DEN BROEK, MD, department of infectious diseases A S LAMPE, MD, PHD, department of medical microbiology G A M BERBÉE, member of infection control committee J THOMPSON, MD, PHD, department of infectious diseases R P MOUTON, MD, PHD, department of medical microbiology

Correspondence to: Dr van den Broek. recommended by the Dutch Working Party on the Standardisation of Sensitivity Tests (WRG, 1981). Phage typing and biotyping (API-Staph, API System SA, Montalieu, France) were performed elsewhere.

\section{The epidemic and results}

During October and November 1981 prosthetic valve endocarditis caused by a strain of Staph epidermidis with unusual resistance to antibiotics (hereinafter called strain I) was diagnosed in four patients at this hospital. Early in October Staph epidermidis had been isolated from the blood of two patients (cases 1 and 2; figure) in the department of thoracic surgery. This strain was resistant to penicillin $G$, methicillin, cephalothin, chloramphenicol, sulphamethoxazole, and rifampicin and sensitive to erythromycin, tetracycline, kanamycin, vancomycin, clindamycin, fusidic acid, and trimethoprim. In case 1 the aortic valve had been replaced by a Björk-Shiley prosthesis on 1 September. At the end of that month Staph epidermidis was grown in several blood cultures. On 4 October a second operation, performed because of dehiscence of the prosthesis, showed an abscess in the anulus fibrosus extending to the ventricular septum and an aneurysm of the ascending aorta. The same strain of Staph epidermidis was cultured from the abscess. A Dacron aortic graft and a Björk-Shiley prosthesis were implanted. On 19 October the patient died of tamponade of the heart.

In case 2 the aortic and mitral valves had been replaced by Björk-Shiley prostheses in June 1981. In the postoperative period a large number of blood cultures had included three growing strain I. Since there were no signs of endocarditis, however, the importance of this was missed. In October heart failure due to dehiscence of the mitral valve prosthesis led to reoperation, and Staph epidermidis strain I was cultured from this prosthesis and the blood.

After the infection control committee started to investigate the possibility of a relation between these two cases two more cases of endocarditis caused by strain I occurred (cases 3 and 4; figure). One of these patients (case 3 ) had been given a Dacron graft for an aneurysm of the ascending aorta and a Björk-Shiley prosthesis on 20 October. On the sixth and seventh postoperative days strain I was cultured from his blood. Antimicrobial treatment was started but one month later he died in a cachectic state with venous thrombosis of the right leg despite adequate anticoagulant treatment, obstruction icterus, and anuria. Necropsy showed carcinoma of the pancreas with multiple metastases. In case 4 the aortic valve was replaced by a BjörkShiley prosthesis on 9 November. Two days later cultures of a sample taken from the heart-lung machine during operation and cultures of the patient's blood yielded Staph epidermidis strain I. Antimicrobial treatment was started. On 27 December reoperation for dehiscence of the prosthesis was

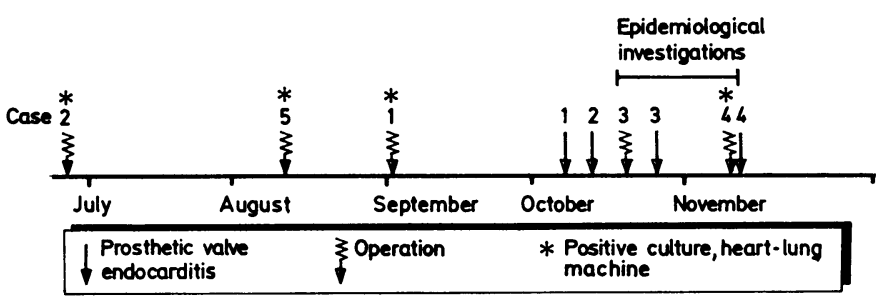

Temporal course of epidemic of prosthetic valve endocarditis caused by strain I Staph epidermidis. 
required. Multiple abscesses and a mycotic aneurysm were found, Staph epidermidis strain I being cultured from both.

\section{EPIDEMIOLOGICAL. INVESTIGATIONS}

As soon as the cultures from cases 1 and 2 had shown Staph epidermidis with the same patterns of resistance and sensitivity to antibiotics (strain I), all the strains of Staph epidermidis isolated since August 1981 from patients in the department of thoracic surgery were re-examined. In cases 1 and 2 strain I was found to have been isolated from cultures of samples taken from the heart-lung machines at the end of the first operation. A fifth patient (figure) was found; in this case Staph epidermidis strain I had been cultured from the sample taken from the heart-lung machine at the end of the operation ( 8 August) and also four days after the operation from a catheter removed from the left atrium. This patient had no signs of infection three months after operation.

To check that the strains of Staph epidermidis from the five patients were identical typing was performed. All five showed the same biotype (APIStaph 6702113) and phage type (U $16(\mathrm{H}))$.

The positive culture of samples from the heart-lung machines in four of the five cases suggested that contamination had occurred during the operation. Review of the surgical teams in all five cases showed that only one person, a surgeon, had been present at all of the operations. Cultures of material from the forehead, nose, and hands of this surgeon, the other 20 members of the operating teams, and the three members of the surgeon's immediate family were set up on blood agar containing chloramphenicol and rifampicin to select for strain I. Strain I was isolated only from the surgeon, from forehead, nose, and hands, and biotyping and phage typing confirmed that it was identical with the strain found in the patients. After the surgeon stopped performing cardiac valve operations there were no further cases of endocarditis caused by Staph epidermidis strain I.

Because the hands of the surgeon were a potential source of infection cultures were taken each hour during a coronary bypass operation performed by him by printing as large as possible a surface of the hands and especially the fingertips on contact plates. Before he washed his hands no Staph epidermidis strain I was isolated, but immediately after he had washed for four minutes with povidone-iodine (Betadine scrub) two colonies were cultured from the right hand. Samples taken during the first two hours of the operation were negative, but after the third hour 18 colonies of strain I were cultured from the right hand, showing that recolonisation of the skin had occurred within about three hours of starting the operation.

\section{TREATMENT OF CARRIER STATE}

Elimination of Staph epidermidis strain I from the surgeon's skin proved to be very difficult. Inspection of the skin showed no abnormalities. In November 1981 he was treated for 10 days, including daily washing of the whole body with povidone-iodine, application of an ointment containing chlorhexidine and neomycin in the nares, hygienic measures such as dry cleaning of personal clothes and blankets, thorough cleaning of utensils (shaving apparatus, comb), and administration of erythromycin stearate 500 $\mathrm{mg}$ four times a day. After completion of this treatment recolonisation of the skin was attempted with an avirulent strain of Staph epidermidis (2504-1; sensitive to methicillin, resistant to chloramphenicol, sensitive to rifampicin) by bathing the surgeon in water containing about $100 \times 10^{6}$ bacteria of the donor strain per 1 and by application of the strain in the nares.

Cultures of samples from the forehead, nose, and hands set up a few days later and after a month still grew Staph epidermidis strain I. A second attempt with the same regimen in January 1982 also failed. A wait and see policy was then followed in the hope that strain I would disappear spontaneously. The surgeon resumed coronary bypass surgery but was not allowed to do operations including implantation of prosthetic material. In December 1982 cultures from his skin still yielded Staph epidermidis strain I. For a third attempt at recolonisation the donor strain was an antibiotic producing Staph epidermidis (S6+, originally isolated by Professor S Selwyn, University of London), which was applied twice daily for five days as a cream containing about $100 \times 10^{6}$ bacteria per $g$ on the forehead, hands, and groins and in the nares. Strain I was found only in the first samples, taken shortly after this treatment, whereas the donor strain was cultured throughout the first three months. After April 1983 neither the epidemic nor the donor strain was isolated from the surgeon's skin. In July 1983 he resumed performing all types of open heart operation and no infectious problems developed.

\section{SECOND CLUSTER OF CASES WITH SINGLE STRAIN OF STAPH EPIDERMIDIS}

Retrospective examination of strains of Staph epidermidis cultured between August and November 1981 from patients in the department of thoracic surgery disclosed a second cluster of six patients with strains that were identical in antibiotic resistance but different from strain I (hereinafter called strain II). Biotyping (API-Staph 6704112) and phage typing (5, 12, $14,16,20)$ confirmed that a single strain was present in these six patients.

Four of the patients had received a cardiac valve prosthesis and the other two had undergone coronary bypass surgery. In three patients strain II was isolated from the heart-lung machine, in one from a blood culture, in one from an intravenous catheter, and in one from the sternal wound. None of the patients had signs or symptoms of infection. Epidemiological investigations showed that these patients too had been operated on by the same surgeon, although not the one responsible for the strain I infections. Strain II was found on the skin of the second surgeon. Because there was no evidence that strain II was pathogenic no action was taken.

\section{Discussion}

Contamination of the surgical site by bacterial skin flora occurs in a high proportion of open heart operations. ${ }^{2}$ The bacteria are thought to reach the wound directly from the patient or from the air. The skin of both the patient and the operating room personnel has been implicated as the source of this contamination. Our findings show that a surgeon may carry a virulent strain of Staph epidermidis and may contaminate the wound during operation. In the first cluster of cases we found that after routine preoperative handwashing the skin of the surgeon's hands was recolonised by Staph epidermidis within the first three hours of starting a long operation. Since gloves are frequently punctured during implantations of heart valves it seems likely that contamination of the surgical site occurred from the hands of the surgeon through puncture holes in his gloves.

Contamination of the wound by Staph epidermidis is not always followed by infection. In the epidemic of prosthetic valve endocarditis caused by strain I four of the five contaminated patients developed an infection as against none of the six patients contaminated by strain II. This disparity might be explained by differences in virulence between the two strains. Epidemics of infection with a single strain of Staph epidermidis have been described in patients undergoing cardiac surgery. ${ }^{4}$ An epidemic with a single person as the source, however, has been reported only once-namely, in three patients given coronary bypass surgery in whom the sternal wound became infected by a strain of Staph epidermidis originating from a resident who was a carrier of this strain.

Carriage of virulent Staph epidermidis is rarely recognised either as a hazard or as an important factor in the epidemiology of nosocomial infection but may have serious consequences.

We thank Dr E E Stobberingh, of the University of Limburg, Maastricht, The Netherlands, for phage typing and biotyping strains I and II; and Professor W C Noble, of the University of London, for supplying strain S6+ Staph epidermidis.

\section{References}

1 Speller DCE, Mitchell RG. Coagulase negative staphylococci causing endocarditis after cardiac surgery. I Clin Pathol 1973;26:517-22.

2 Kluge RM, Calia FM, McLaughlen JS, Hornick RB. Sources of contamination in open heart surgery. FAMA 1974;230:1415-8.

3 Blakemore WS, McGarrily GJ, Thurer RJ, Wallace HW, MacVaugh H III, Correll LL. Infection by air-borne bacteria with cardiopulmonary bypass. Surgery 1971;70:830-8.

4 Archer GL, Vishniavsky N, Stiver HG. Plasmid pattern analysis of staphylococcus epidermidis isolates from patients with prosthetic valve endocarditis. Infect Immun 1982;35:627-32.

5 Maki D, Zilz M, Alvarado C, Robbins J, Parisi J. Methicillin-resistant Staph epidermidis surgical wound infections linked to a chronic carrier. Abstracts of International conference of antimicrobial agents and chemotherapy 1982; No 566:166.

\section{(Accepted 10 fuly 1985)}

Hairdressers often put pins and combs in their mouths. Could acquired immune deficiency syndrome be spread in this way from hairdresser to client?

I believe that it is most unlikely that the acquired immune deficiency syndrome could be acquired by clients from hairdressers as a result of the latte holding pins or combs in their mouths. The present evidence that health service staff have not contracted the syndrome from patients, despite an increasing number of reports of needle stick injuries, is reassuring in this respect.-A M GEDDES, professor of communicable and tropical diseases, Birmingham. 